\title{
Evaluation Comparee De La Malnutrition Des Enfants De Moins De 5 Ans Par Des Indices Anthropometriques En Republique Democratique Du Congo
}

\author{
Djomo Lokanga Godefroy \\ Département des Sciences de la Population et du Développement, \\ Université de Kinshasa
}

Doi:10.19044/esj.2021.v17n7p154

Submitted: 19 December 2020

Accepted: 08 January 2021

Published: 28 February 2021
Copyright 2021 Author(s)

Under Creative Commons BY-NC-ND

4.0 OPEN ACCESS

Cite As:

Djomo L.G. (2021). Evaluation Comparee De La Malnutrition Des Enfants De Moins De 5 Ans Par Des Indices Anthropometriques En Republique Democratique Du Congo. European Scientific Journal, ESJ, 17(7), 154. https://doi.org/10.19044/esj.2021.v17n7p154

\section{Résumé}

La dénutrition des enfants est conventionnellement évaluée par trois indicateurs de l'état nutritionnel constitués par des indices de mesure anthropométrique, à savoir l'émaciation (poids/taille), le retard de croissance (taille/âge) et l'insuffisance pondérale (poids/âge). Cependant, ces indicateurs sont critiqués de sous-estimer la prévalence de la malnutrition étant donné que ceux-ci se chevauchent. C'est ainsi qu'un autre indicateur a émergé à savoir l'indice composite d'échec anthropométrique (CIAF). Vu que le CIAF ne donne pas les formes de malnutrition que présentent les enfants dans leur ensemble, il a été proposé l'indice de l'émaciation, l'indice de retard de croissance et l'indice de l'insuffisance pondérale pour fournir le profil clinique de la dénutrition totale. L'objectif de cette étude est d'évaluer l'état nutritionnel des enfants à travers ces indices susmentionnées en utilisant les données MICS 2017-2018 de la RD Congo et de comparer ceux-ci avec des indicateurs conventionnellement utilisés. Les résultats révèlent qu'en RD Congo, la prévalence de la dénutrition des enfants de moins de 5 ans par le CIAF est de 47,3\%. Dans cette prévalence, la proportion de l'indice de l'émaciation, l'indice de retard de croissance et l'indice de l'insuffisance pondérale est respectivement de $0,135,0,867$ et 0,488 . L'étude montre que les indicateurs conventionnels sous-estiment la dénutrition des enfants et peuvent également modifier l'ordre des milieux les plus préoccupants. 
L'étude suggère que ces indices soient complémentaires aux indicateurs conventionnels afin d'élargir le champ de vision des décideurs dans leurs interventions pour lutter de manière plus stratégique contre la malnutrition.

Mots clés : Indice Composite D'échec Anthropométrique, Indice De L'émaciation, Indice De Retard De Croissance, Indice D'insuffisance Pondérale, Malnutrition

\section{Assessment of Malnutrition of Children Under 5 by Anthropometric Indices in the Democratic Republic of Congo}

\section{Djomo Lokanga Godefroy}

Département des Sciences de la Population et du Développement, Université de Kinshasa

\section{Abstract}

The undernutrition of children is conventionally assessed by three indicators of nutritional status constituted by anthropometric measurement indices, namely wasting (weight/height), stunting (height/age) and underweight (weight/age). However, these indicators are criticized for underestimating the prevalence of malnutrition since these overlap. Thus, a new indicator was proposed, namely the Composite Index of Anthropometric Failure (CIAF). Since the CIAF does not provide the forms of malnutrition that children as a whole present, the wasting index, stunting index and underweight index were proposed to provide the clinical profile of total undernutrition. The objective of this study is to assess the nutritional status of children through these new indices mentioned above using the MICS 2017-2018 data from DR Congo and to compare them with conventionally used indicators. The results reveal that in DR Congo, the prevalence of undernutrition of children under 5 by the CIAF is $47.3 \%$. In this prevalence, the proportion of the wasting index, the stunting index and the underweight index are respectively $0.135,0.867$ and 0.488 . The study shows that conventional indicators underestimate child undernutrition and can also change the order of the settings of greatest concern. The study suggests that these indices are complementary to conventional indicators in order to broaden the field of vision of decision-makers in their interventions to fight more strategically against malnutrition.

Keywords: Composite Index Of Anthropometric Failure, Wasting Index, Stunting Index, Underweight Index, Malnutrition 


\section{Introduction}

La malnutrition touche plus d'un enfant sur trois dans le monde et elle est plus prononcée dans les pays en voie de développement particulièrement dans ceux de l'Asie du sud et de l'Afrique Subsaharienne (UNICEF, 2019). En République Démocratique du Congo (RD Congo), selon la dernière Enquête à Indicateurs Multiples (MICS, 2017-2018), la prévalence des enfants âgés de moins de 5 ans atteints de l'émaciation, du retard de croissance et de l'insuffisance pondérale est respectivement de 7\%, $42 \%$ et $23 \%$. Ces proportions restent très similaires à celles de la dernière Enquête Démographique et de Santé (EDS, 2013-2014) qui indiquent 8\%, $43 \%$ et $23 \%$ pour chacun des indices susmentionnés. Cette prévalence est de loin supérieure à celle d'une population en bonne santé selon les normes de l'Organisation Mondiale de la Santé (OMS) qui veut que la prévalence du retard de croissance dans une population en bonne santé et bien nourris n'excède pas 2,3\% (OMS, 2008).

Par ailleurs, des travaux menés autour de la malnutrition ont révélé que les indices conventionnels par lesquels ont été calculés les indicateurs de l'état nutritionnel des enfants tels que présentés ci-haut, sont sujet à des lacunes qui peuvent réduire le champ de vision des décideurs censés lutter contre la malnutrition notamment du fait que la probabilité associée à la morbidité et à la mortalité des enfants malnutris diffère selon les formes de malnutrition que présentent les enfants (McDonald et al., 2013 ; Nandy et al., 2005; Nandy et Svedberg, 2012). Ces indicateurs conventionnels sont critiqués de sous-estimer la prévalence de la malnutrition, car ils se chevauchent alors que chacun d'eux se constituent des individus ayant des formes de malnutrition distinctes. En effet, certains enfants peuvent être simultanément en retard de croissance et en insuffisance pondérale sans être émaciés; émaciés sans être en retard de croissance ou en insuffisance pondérale; et aussi en insuffisance pondérale sans pour autant souffrir d'une autre forme de malnutrition (Nandy et al., 2005). D'un autre côté, l'indice composite (l'insuffisance pondérale) censé refléter l'état nutritionnel global ne se calcule pas par la somme des enfants atteints du retard de croissance et de ceux atteints de l'émaciation. N'étant pas la somme des enfants malnutris, on peut dire que l'insuffisance pondérale reflète mal l'ampleur globale du problème nutritionnel dans une population. De plus, il a également été démontré que dans l'évaluation des progrès accomplis dans le suivi de la malnutrition, "l'insuffisance pondérale peut exagérer l'ampleur du changement $»$ ( Nandy et Mianda, 2008:1965).

Face à ces lacunes, Svedberg (2000) construit un indicateur agrégé, appelé indice composite de défaillance nutritionnel (Composite Indice of Anthropometric Faillure (CIAF)) qui permet de palier au problème de sousestimation des enfants sous-alimentés et en même temps de contourner le 
chevauchement observé dans les indices conventionnels. Le CIAF a l'avantage de refléter une meilleure image de l'état nutritionnel des enfants puisqu'il présente d'une part les enfants normaux (ceux qui n'ont aucun échec anthropométrique ou aucune forme de malnutrition) et les enfants malnutris (ceux qui présentent une ou plusieurs formes de malnutrition). En bref, le CIAF met en évidence l'ampleur du problème de la dénutrition des enfants et peut de ce fait répondre de manière globale à la question « combien d'enfants sont malnutris dans une population ?».

En dépit de tous ces avantages, le besoin d'informations supplémentaires a mis en lumière quelques limites reportées au CIAF (Bhattacharya, 2006). Celui de savoir la contribution respective de l'ampleur de l'émaciation, du retard de croissance et de l'insuffisance pondérale dans le CIAF qui ne présente que la situation globale. Car dans l'évaluation de la malnutrition, une classification clinique des formes de malnutrition reste très importante pour guider les actions des spécialistes. Pour remédier à cela, Bose et Mandal (2010) ont proposé de nouveaux indices, à savoir l'indice de retard de croissance, l'indice de l'émaciation et l'indice de l'insuffisance pondérale. Ces indices ne sont pas similaires à ceux conventionnellement utilisés du fait qu'ils sont calculés à partir du CIAF et reflètent ainsi la part de chacune des formes de malnutrition parmi tous les enfants malnutris.

De plus, à travers des indicateurs désagrégés tirés du CIAF, on peut notamment identifier quel type de défaillance anthropométrique est associé au risque élevé de morbidité (Nandy et al., 2005 ; Nandy et Svedberg, 2012) ou de mortalité des enfants (McDonald et al., 2013). Ce qui est une source d'information non négligeable pour des programmes et interventions de réduction du risque de morbidité et de mortalité avec la malnutrition comme cause sous-jacente.

Ainsi, l'objectif de la présente étude est d'évaluer l'état nutritionnel des enfants de moins de 5 ans en RD Congo en utilisant l'ensemble des indicateurs qui ont émergé dans la littérature et de comparer ces indicateurs avec ceux conventionnellement utilisés.

\section{Methodologie}

Les données utilisées dans cette étude proviennent du MICS 20172018. Cette enquête est la plus récente, d'une couverture nationale et représentative de la population de la R.D. Congo. L'enquête a sélectionné dans tous les 26 provinces de la RD Congo 21.477 enfants (49,5\% garçons et $50,5 \%$ filles) âgés de 0 à 59 mois. Les enfants qui avaient au moins une information manquante ou des indices aberrants ont été exclus de l'analyse. Ce qui laisse un total de 21453 enfants (99,9\% de l'échantillon du départ). Chaque enfant sélectionné dans l'échantillon a été pesé et mesuré par les enquêteurs pour recueillir leurs données anthropométriques. Les analyses ont 
été effectuées via le logiciel SPSS où les observations ont été pondérées par la variable du poids de l'échantillon des enfants de moins de 5 ans nommée chweight.

Pour évaluer l'état nutritionnel des enfants, le CIAF sera construit et ensuite les trois indices proposés par Bose et Mandal (2010) seront calculés afin de montrer la part de contribution du retard de croissance, de l'émaciation et de l'insuffisance pondérale dans le nouvel indicateur agrégé. $\mathrm{Au}$ même titre que les trois indices conventionnels, le CIAF se construit à travers le Z-score ou l'unité d'écart-type trouvé par des mesures anthropométriques des enfants (Poids, taille et âge) selon la formule suivante (OMS, 1995) :

$$
\mathrm{Z}-\text { scores ou unité d'écart }- \text { type }=\frac{X-\text { Médiane de la population de référence }}{\text { Ecart }- \text { type de la population de référence }}
$$

- $\quad$ où X est la valeur observée (l'âge, le poids ou la taille des enfants)

Pour tout indice anthropométrique calculé, lorsque la valeur de l'écarttype est strictement inférieur à -2 , le sujet est considéré comme atteint de la malnutrition. La population de référence avec laquelle a été calculé ces indices provient des nouvelles normes de croissance et de nourrisson de l'OMS en 2006, qui pour cette fois intégrait aussi dans la population de référence les pays en voie de développement. Ces pays sont les suivants : le Brésil, les Etats-Unis, le Ghana, l'Inde, la Norvège et l'Oman

Précisons qu'à l'origine, le CIAF tel que construit par Svedberg (2000) était structuré en six groupes de $(\mathrm{A}$ à $\mathrm{F})$. Le groupe $\mathrm{A}$, est constitué des enfants ne souffrant d'aucune défaillance anthropométrique et les groupes restant (de B à F) sont constitués des enfants dont la taille et le poids pour leur âge sont inférieurs aux normes de croissance et souffrants ainsi d'au moins une forme de malnutrition. Cependant, Nandy et al. (2005) ont identifié une septième classification d'enfants malnutris. Ils l'ont intitulé le groupe $\mathrm{Y}$. Ce groupe est constitué des enfants ne souffrant que de l'insuffisance pondérale, c'est-à-dire des enfants qui n'ont qu'un poids insuffisant mais ne sont pas émacié et n'ont pas de retard de croissance. Cette classification présente des formes de malnutritions qui ne se chevauchent pas, elles sont mutuellement exclusives (tableau 1). C'est sur cet ensemble de toutes les formes de malnutrition que se composera le CIAF (indicateur agrégé) en additionnant les groupes d'enfants qui présentent au moins une forme de malnutrition; ce qui n'était pas le cas pour des indicateurs conventionnels. Autrement dit, le CIAF se constitue de la somme de l'effectif de tout le « oui » tel qu'il est figuré dans le tableau 1. 
Tableau 1. Classification selon les défaillances anthropométriques

\begin{tabular}{clccc}
\hline Groupe & Description & Emaciation & $\begin{array}{c}\text { Retard de } \\
\text { croissance }\end{array}$ & $\begin{array}{l}\text { Insuffisance } \\
\text { Pondérale }\end{array}$ \\
\hline A & Pas d'échec & Non & Non & Non \\
B & Emaciation seulement & Oui & Non & Non \\
C & Emaciation et Insuffisance pondérale & Oui & Non & Oui \\
D & Emaciation, Retard de croissance et Insuffisance pond. & Oui & Oui & Oui \\
E & Retard de croissance et Insuffisance pondérale & Non & Oui & Oui \\
F & Retard de croissance uniquement & Non & Oui & Non \\
Y & Insuffisance pondérale uniquement & Non & Non & Oui \\
\hline
\end{tabular}

*Classification selon Nandy et al. (2005)

Pour évaluer la contribution respective de l'indice du retard de croissance, l'indice de l'émaciation et de l'indice de l'insuffisance pondérale dans le CIAF, chaque indice conventionnel est divisé par le CIAF (Bose et Mandal, 2010), soit :

- Indice de retard de croissance $=$ retard de croissance $/$ CIAF

- Indice de l'insuffisance pondérale = insuffisance pondérale / CIAF

- Indice de l'émaciation = émaciation / CIAF

Les valeurs trouvées ici n'ont pas d'unité, étant un rapport, on comprend que plus la valeur est élevée, plus la gravité de l'indice l'est également. Toutefois, il est encouragé d'interpréter ces valeurs comme des pourcentages.

\section{Resultats}

\section{Prévalence de la Malnutrition}

Les résultats du tableau 2 montrent que 23,1\% d'enfant souffrent de la malnutrition aigüe et $41 \%$ d'enfant souffrent de la malnutrition chronique. L'insuffisance pondérale comme indicateur agrégé est de 23,1\%. Et le CIAF (tableau 3) montre qu'en RD Congo, dans l'ensemble, 47,3\% présente une ou plusieurs formes de malnutrition. De manière désagrégée, cet indicateur composite indique que les enfants souffrant uniquement de l'émaciation, du retard de croissance et de l'insuffisance pondérale (groupe B, F et Y) sont respectivement de $2,1 \%, 22 \%$ et $2,1 \%$. On remarque qu'en se fiant uniquement à l'indice conventionnel agrégé (l'insuffisance pondérale) les enfants du groupe B et $\mathrm{F}$ qui présentent un total $24,1 \%$ de tous les enfants, auraient été mal classés car ils ne présentent pas des signes d'insuffisance pondérale. Les enfants théoriquement les plus vulnérables, c'est-à-dire ceux qui présentent simultanément tous les trois formes de malnutrition (groupe D) sont de l'ordre de 2,3\%. 
Tableau 2. Prévalence de la malnutrition des enfants de moins 5 ans selon les types des indicateurs

\begin{tabular}{lc}
\hline Indices & Nombre d'enfant $(\boldsymbol{\%})$ \\
\hline Emaciation & $1505(6,4)$ \\
Retard de croissance & $9264(41)$ \\
Insuffisance pondérale & $5150(23,1)$ \\
CIAF & $\mathbf{1 0 6 3 1 ( 4 7 , 3 )}$ \\
\hline
\end{tabular}

Tableau 3. Distribution des statuts nutritionnels des enfants de moins de 5 ans selon le CIAF

\begin{tabular}{clc}
\hline Groupe & Description & Nombre d'enfant (\%) \\
\hline A & Pas d'échec & $10822(52,7)$ \\
B & Emaciation seulement & $447(2,1)$ \\
C & Emaciation et Insuffisance pondérale & $465(2)$ \\
D & Emaciation, Retard de croissance et Insuff; pondérale & $591(2,3)$ \\
E & Retard de croissance et Insuffisance pondérale & $3637(16,7)$ \\
F & Retard de croissance uniquement & $5034(22)$ \\
Y & Insuffisance pondérale uniquement & $457(2,1)$ \\
Total & & 21453 \\
\hline
\end{tabular}

Suivant les caractéristiques des enfants (le tableau 4), les résultats révèlent que dans l'ensemble les garçons souffrent plus de la malnutrition que les filles. La sous-estimation du niveau de la malnutrition reprochée aux indices conventionnels du fait que ces derniers se chevauchent, parait plus flagrante lorsque les données sont ventilées selon certaines caractéristiques des enfants. Alors que l'indicateur conventionnel agrégé est de 21,4\% pour les filles, le CIAF s'élève à plus de double de ce pourcentage $(45,6 \%)$. Et les tendances similaires s'observent sur toutes les caractéristiques restantes. On constate également que la prévalence de la malnutrition augmente avec l'âge des enfants pour tous les indicateurs, exception fait de l'émaciation. Pour ce qui est du CIAF selon milieu de résidence, en dépit du fait que l'écart entre le milieu urbain et le milieu rural est de 21 points de pourcentage $(34,6 \%$ contre 55,3\%), la dénutrition totale des enfants reste élevée dans ces deux types de milieu.

Tableau 4. Prévalence des indicateurs de la malnutrition selon les caractéristiques des enfants

\begin{tabular}{lcccc}
\hline $\begin{array}{c}\text { Caractéristiques } \\
\text { des enfants }\end{array}$ & Emaciation & $\begin{array}{c}\text { Retard de } \\
\text { croissance }\end{array}$ & $\begin{array}{l}\text { Insuffisance } \\
\text { pondérale }\end{array}$ & CIAF \\
\hline Sexe & & & & \\
Masculin & 7,1 & 46,8 & 26,6 & 53,6 \\
Féminin & 5,8 & 39,6 & 21,4 & 45,6 \\
Âge (en mois) & & & & \\
0-11 & 8,3 & 23,4 & 15,7 & 32,4 \\
$12-23$ & 8,4 & 40,9 & 22,1 & 48 \\
$24-35$ & 5,3 & 47,9 & 24,8 & 52,7 \\
$36-47$ & 5,1 & 46,2 & 24,9 & 50,7 \\
\hline
\end{tabular}




\begin{tabular}{lcccc}
\hline $\begin{array}{c}48-59 \\
\text { Milieu de Résidence }\end{array}$ & 5,2 & 46,5 & 28 & 52,4 \\
$\quad$ Urbain & 5,5 & 28,50 & 14,7 & 34,60 \\
Rural & 7,1 & 48,90 & 28,4 & 55,30 \\
\hline
\end{tabular}

Le graphique 1 illustre comment se répartit l'ampleur de la malnutrition à travers différents indices dans toutes les provinces de la R.D. Congo. De celle-ci, deux constats peuvent être faits. Premièrement on remarque que les intensités données par le CIAF sont plus proches de celles données par le retard de croissance que celles de l'insuffisance pondérale alors que ce dernier est utilisé comme indicateur agrégé. Deuxièmement, on constate que classer les provinces selon l'ordre de leurs prévalences de malnutrition donne des résultats différents lorsque l'on compare le CIAF et l'indicateur conventionnel agrégé (l'Insuffisance pondérale). $\mathrm{Si}$ on s'intéresse au total des enfants présentant une ou plusieurs formes de malnutrition (CIAF), la province de Kwango et de Kasaï Centrale sont les plus alarmantes avec respectivement $60,5 \%$ et $57,3 \%$ des enfants malnutris (ce classement ne varie que légèrement lorsqu'on considère le retard de croissance). L'insuffisance pondérale à son tour, nous laisse voir un autre tableau avec la province du Nord Ubangi et la province du Kwango comme les plus alarmantes avec respectivement $13 \%$ et $10,9 \%$ des enfants malnutris.

Cependant, la ville de Kinshasa (capitale) enregistre la plus faible prévalence de la malnutrition en utilisant le CIAF (22\%) alors qu'en utilisant l'insuffisance pondérale, c'est la province du Sud Kivu qui enregistre les moins d'enfants malnutris $(2,6 \%)$. En considérant ce dernier indicateur, la ville de Kinshasa où on s'attend enregistrer les moins d'enfants souffrant de la malnutrition, arrive après 7 provinces (Tanganyika, Maniema, Bas Uele, Tshopo, Nord Kivu, Sud Ubangi, Haut Katanga) avec une prévalence de $5,4 \%$.

Pourcentage

Graphique 1. Prévalence de la malnutrition selon les provinces

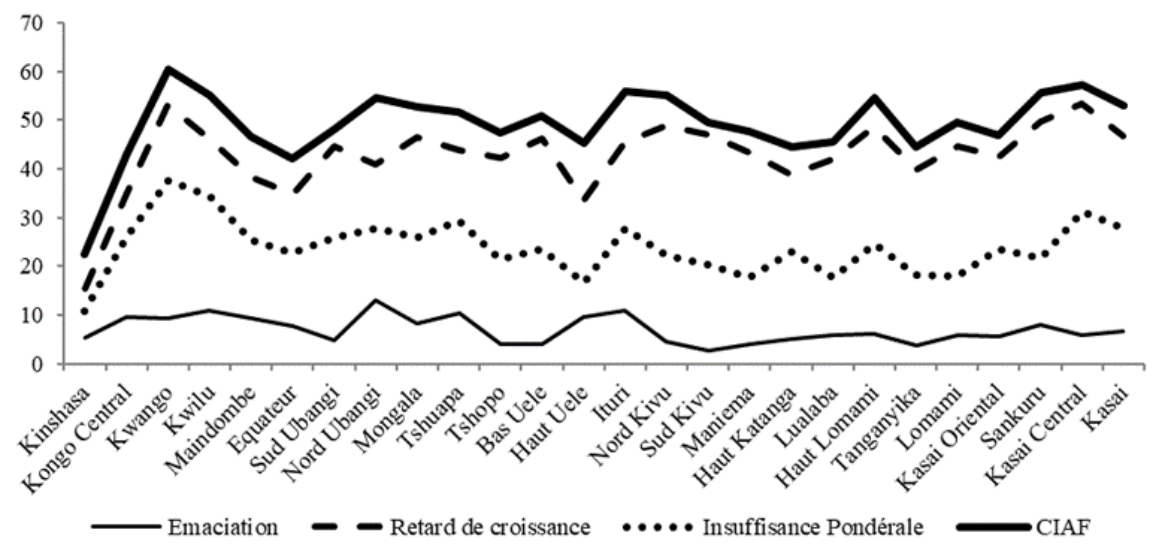




\section{Identification des formes de malnutrition dans le CIAF}

Le CIAF a montré qu'en RD Congo au totale près de la moitié de tous les enfants sont malnutris. Le profil clinique de cette prévalence, c'està-dire la part de l'émaciation, du retard de croissance et de l'insuffisance pondérale dans cet indicateur se présente dans le tableau 5. Etant donné que les valeurs trouvées peuvent également être interprétées comme des pourcentages (Bose et Mandal, 2010, p. 134), on peut dire que dans le total de 47,3\% des enfants malnutris, la prévalence de l'émaciation est de 13,5\%, celle du retard de croissance est de $86,7 \%$ et $48,8 \%$ pour de l'insuffisance pondérale.

Tableau 5. Profil nutritionnel dans le CIAF

\begin{tabular}{lc}
\hline Indices & Valeurs \\
\hline Indice de l'Emaciation = Emaciation/CIAF & 0,135 \\
Indice du Retard de croissance = Retard decroissance/CIAF & 0,867 \\
Indice de l'Insuffisance pondérale = Insuffisance pondérale /CIAF & 0,488 \\
\hline
\end{tabular}

Dans le tableau 6, on note que pour la prévalence de l'émaciation dans le CIAF diminue avec l'augmentation de l'âge des enfants et aussi que la malnutrition chronique est trop élevée selon les différentes caractéristiques où elle peut atteindre jusqu'à plus de 9 enfants sur 10.

Le graphique 2 montre que parmi les enfants malnutris, la proportion des émaciés garde la tendance baissière et le retard de croissance reste toujours aussi élevé. Les données révèlent que la ville province de Kinshasa, qui a souvent présenté des plus faibles prévalences de la malnutrition pour tout indicateur utilisé, possède cette fois la valeur la plus élevée pour l'indice de l'émaciation $(0,24)$. Il faut garder à l'esprit que ces valeurs sont calculées à partir du CIAF, c'est-à-dire que la haute prévalence de l'émaciation observée à Kinshasa est tirée de la faible proportion d'enfants souffrant de la malnutrition dans cette ville. Ces résultats suggèrent que la qualité de vie urbaine, les meilleurs soins de santé nutritionnels et différents autres programmes et prises en charge présents dans la ville, montrent bien leurs efficacités dans la luttant contre la malnutrition vu les faibles valeurs de l'indice de retard de croissance et de l'indice de l'insuffisance pondérale. Les avantages du milieu urbain empêchent la malnutrition dans sa forme aigüe de se prolonger à une durée plus longue pour se transformer en malnutrition chronique. Ce qui n'est pas le cas dans les autres milieux plus précaires où la malnutrition se prolonge plus facilement dans sa forme chronique jusqu'à atteindre plus de $90 \%$ d'enfants. 
Tableau 6. Valeurs de l'Indice de l'Emaciation, Indice de Retard de croissance et de l'indice d'insuffisance pondérale dans le CIAF selon les caractéristiques des enfants

\begin{tabular}{lccc}
\hline $\begin{array}{c}\text { Caractéristiques } \\
\text { des enfants }\end{array}$ & Emaciation & $\begin{array}{c}\text { Retard de } \\
\text { croissance }\end{array}$ & $\begin{array}{c}\text { Insuffisance } \\
\text { pondérale }\end{array}$ \\
\hline $\begin{array}{c}\text { Sexe } \\
\text { Masculin }\end{array}$ & 0,139 & 0,873 & \\
$\quad \begin{array}{l}\text { Féminin } \\
\text { Agge (en mois) }\end{array}$ & 0,134 & 0,864 & 0,508 \\
$0-11$ & 0,256 & 0,722 & 0,485 \\
$12-23$ & 0,175 & 0,852 & 0,460 \\
$24-35$ & 0,101 & 0,909 & 0,471 \\
$36-47$ & 0,101 & 0,911 & 0,491 \\
$48-59$ & 0,099 & 0,887 & 0,534 \\
Milieu de Résidence & & & \\
Urbain & 0,159 & 0,824 & 0,425 \\
Rural & 0,128 & 0,884 & 0,514 \\
\hline
\end{tabular}

Graphique 2. Valeurs de l'Indice de l'Emaciation, l'Indice du Retard de croissance et de l'Indice de l'Insuffisance pondérale dans le CIAF selon les provinces

Pourcentage

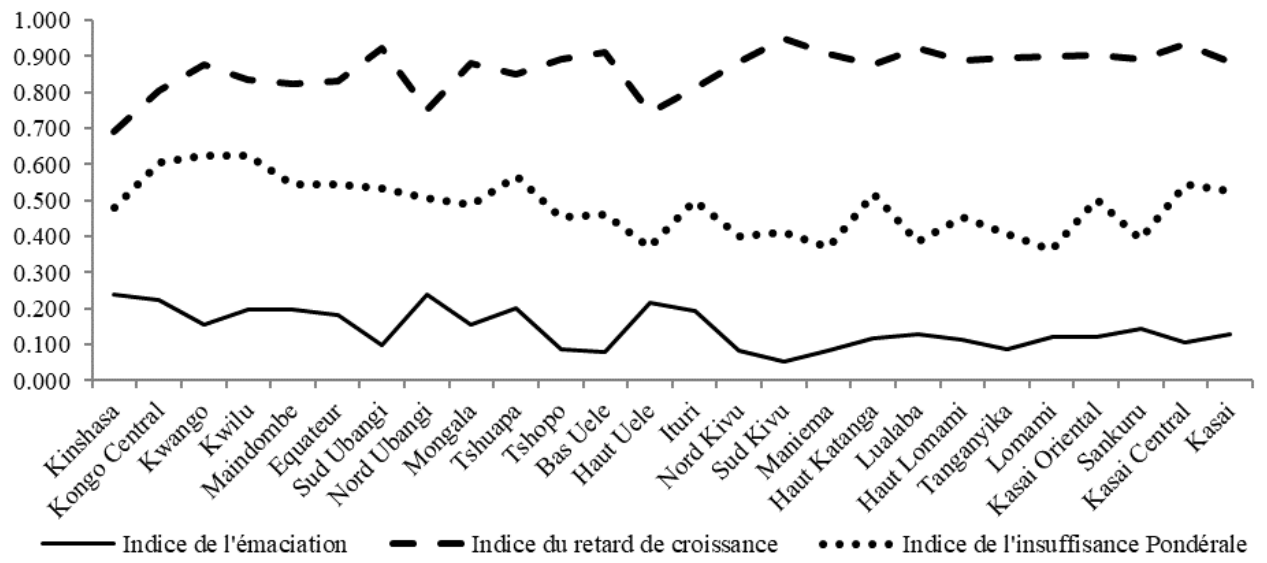

L'image que laisse voir ces trois nouveaux indicateurs est préoccupant car il en ressort que parmi les enfants malnutris, dans 8 provinces (Sud Kivu, Kasaï Central, Sud Ubangi, Lualaba, Bas Uele, Maniema, Kasaï Oriental et Lomami) plus de 9 enfants sur 10 présentent une malnutrition chronique qui traduit une restriction du potentiel de croissance en raison d'une carence nutritionnelle ou une privation alimentaire prolongée. Et cette proportion tourne autour de 8 enfants sur 10 pour toutes les autres provinces restantes à l'exception de Kinshasa (68,9\%), de Haut Uele $(74,3 \%)$ et du Nord Ubangi $(75,2 \%)$. 


\section{Discussion}

Les résultats des indicateurs convergent avec ceux du rapport du MICS 2017-2018 uniquement pour l'émaciation et le retard de croissance. Ceux de l'insuffisance pondérale diffère de ceux du rapport à seulement un point de pourcentage. Le CIAF donne un pourcentage de plus de deux fois supérieur à celui de l'insuffisance pondérale. Cela montre l'ampleur de la sous-estimation des enfants malnutris causée par le chevauchement qui pèse sur l'insuffisance pondérale. Le nombre d'enfants en dénutrition augment avec l'âge, tant pour le CIAF que pour l'insuffisance pondérale. Le contexte des précarités observées en RD Congo entraine de l'insécurité alimentaire et cela explique cette tendance de dénutrition avec l'augmentation de l'âge des enfants. Car le besoin en nutriment est de moins en moins satisfait lorsque les enfants grandissent et demandent des compléments nutritionnels. Des résultats similaires sont observés dans d'autres régions frappées par la malnutrition (Sengupta et al., 2010).

Dans cette étude, l'échec anthropométrique est plus élevé chez les garçons que chez les filles. Ces résultats s'accordent au contexte des pays africains où les mêmes tendances ont été observé dans plusieurs autres pays dans la partie Sub-saharienne (Wamani et al., 2007). L'ordre du classement des provinces ayant les plus d'enfants souffrant de la malnutrition ainsi que celles ayant les moins d'enfants est le même pour l'indicateur conventionnel agrégé et le CIAF mais à des prévalences bien très différentes (quoique cet ordre varie légèrement par la suite). Ces résultats s'accordent avec plusieurs autres études (Dewan, Gupta et Kumar, 2015 ; Seetharaman et al., 2007 ; Mandal et Bose 2009 ; Nandy et al., 2005 ; Nandy et Miranda, 2008) pour affirmer que les indicateurs conventionnels ont tendance à sous-estimer le nombre d'enfants souffrant de la malnutrition. Et ceci peut entraver l'ordre de priorité ou réduire le champ de vision des décideurs étant donné que le CIAF, dans sa forme désagrégé, peut montrer les régions ayant plus d'enfants souffrant simultanément des plusieurs formes de malnutrition. Etant donné que la malnutrition est plus morbide ou plus létale lorsqu'elle se présente simultanément sous plusieurs formes chez le même enfant.

Les information supplementaires fournies dans l'utilisation du CIAF participe à une meilleure évaluation de la malnutrition des enfants. Car cette évaluation serait peut guider les actions et planifications des décideurs qui auront les informations sur la prévalence totale de la dénutrition dans l'ensemble de la population concernée. Aussi, le CIAF a été reconnu pour ces meilleurs perspectives à travers son utilisation dans un corpus de travaux sur la malnutrition menées en Inde (Seetharaman et al., 2007 ; Goswami, 2016 ; Mandal et Bose 2009 ; Shit et al., 2012 ; Nandy et al., 2005 ; Nandy et Svedberg, 2012 ; Dewan, Gupta et Kumar, 2015 ; Ramkuma et al., 2018), en Afrique (Emina, 2009; Nguemo, 2019; Berger, Hollenbeck et Fields- 
Gardner, 2006 ; Mahgoub et Fields-Gardner, 2009) ainsi que dans d'autres régions du continent où sévit le problème de malnutrition des enfants (Dang \& Yan, 2007 ; Harttgen et Misselhorn 2006).

La valeur déduit de l'indice de l'émaciation, de l'indice du retard de croissance et de l'indice de l'insuffisance pondérale à travers l'ampleur de la dénutrition globale était respectivement de 0,135, 0,867 et 0,488. Ces valeurs sont supérieures à celles des indicateurs conventionnels qui renseignent sur les mêmes formes de malnutrition.

Graphique 3. Comparaisons de l'Indice de l'Emaciation, l'Indice du Retard de croissance et de l'Indice de l'insuffisance pondérales des études en Afrique

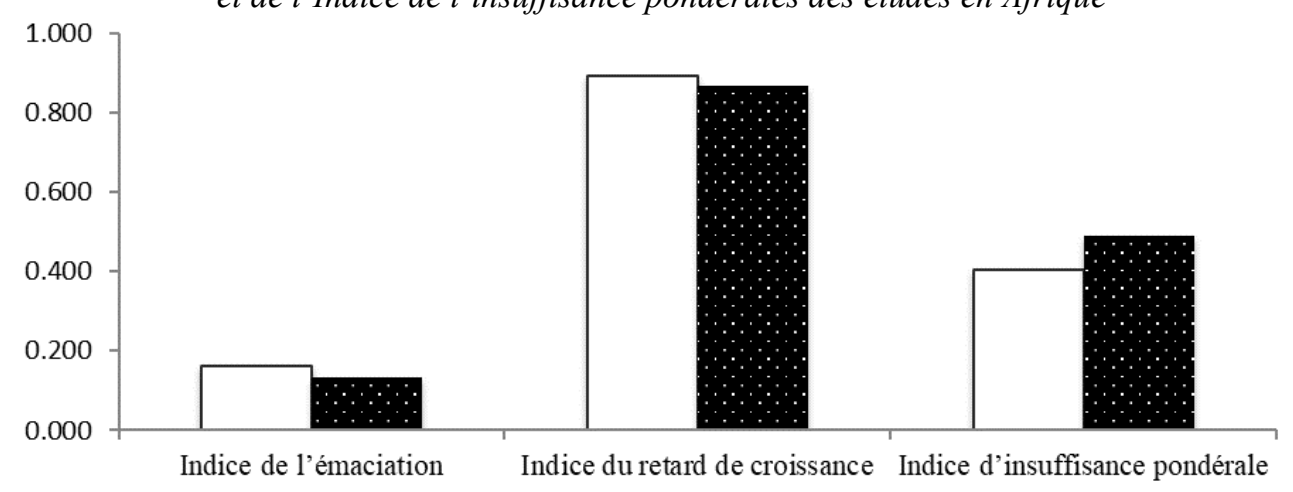

$\square$ Nguemo (2019) n $=5189$

Cette étude $n=21453$

Ces trois indices ont été calculés sur base des résultats d'une récente évaluation effectuée au Cameroun (Nguemo, 2019) sur la malnutrition des enfants des moins de 5 ans afin de les comparer avec ceux de cette étude. Les résultats indiquent que (graphique 3) malgré une prévalence plus élevée du CIAF en RD Congo comparativement à celui du Cameroun $(47,3 \%$ contre $37 \%$ ), l'indice de l'émaciation et l'indice de retard de croissance du Cameroun sont légèrement supérieur à ceux de la RD Congo (respectivement 0,135 contre 0,162 et 0,867 contre 0,892 ). Par contre pour l'indice de l'insuffisance pondérale, les résultats du Cameroun est inférieur à ceux de la RD Congo $(0,405$ contre 0,488$)$. Ces indices calculés à partir du CIAF ne sont pas sujets à des chevauchements et expriment ainsi le fardeau réel des différentes formes des malnutritions qui pèsent sur ces enfants. Ces informations n'étant basées que sur les enfants en dénutrition, elles présentent la prévalence des différentes formes de malnutrition auprès de la totalité enfants malnutris.

Les resultats montrent aussi que l'ordre des provinces les plus préoccupantes change selon que l'on utilise les indicateurs conventionnels ou les nouveaux indices. Cependant, en dépit de ce changement de l'ordre des provinces les plus nécessiteuses, dans l'ensemble, quasiment les mêmes groupes des provinces reviennent dans la liste des plus préoccupantes ainsi 
que dans le groupe des moins touchés par la malnutrition. Ce qui nous amène à nous accorder avec ce que suggèrent d'autres auteurs (Dewan, Gupta et Kumar, 2015 ; Bose et Mandal, 2010), pour appuyer que ces nouveaux indices ne remplacent aucunement les anciens indicateurs, mais ils offrent des informations complémentaires sans chevauchement ou sous-estimation des enfants malnutris.

Les régions figurant en haut de la liste doivent impérativement être prise en compte car la malnutrition chronique qui frappe des enfants durant plus de deux ans, produit des effets négatifs irréversibles pour l'avenir de ces derniers. Et pour le groupe d'enfants les plus vulnérables, ceux qui présentent simultanément plusieurs formes de malnutrition, le CIAF révèle que leur proportion était de près de $22 \%$. En présence de ces informations, en priorisant ce groupe d'enfants, des programmes stratégiques pour réduire la malnutrition pourront montrer plus des dividendes. Car les interventions vont agir en même temps sur la morbidité avec la malnutrition comme cause sousjacente ainsi que sur la mortalité des enfants malnutris.

\section{Conclusion}

La présente étude visait à évaluer et à comparer l'état nutritionnel des enfants de moins de cinq ans en RD Congo à travers différents indices anthropométriques. L'écart entre l'indicateur retenu pour se renseigner de la prévalence de la malnutrition et l'indicateur qui renseigne sur l'ampleur globale de la malnutrition est de 25 points de pourcentage. Cette sousestimation peut représenter des millions d'enfants malnutris vu l'effectif galopant d'enfants en R.D. Congo. Et le CIAF a montré un autre tableau des régions à plus haute prévalence de malnutrition chronique légèrement différent de celui de du retard de croissance conventionnel. Vu l'échec dans la réalisation des Objectifs Millénaire du Développement, les décideurs devraient prendre en compte d'autres suggestions pertinentes, telles que celles que laissent voir ce document, dans le suivi et la prise en charge des enfants en échec anthropométrique. Ceci pourrait participer à combattre plus stratégiquement la malnutrition qui sévit en RD Congo afin d'atteindre la deuxième cible des Objectifs du Développement Durable d'ici 2030.

\section{References:}

1. Berger M, Hollenbeck C \& Fields-Gardner C (2006). "Prevalence of malnutrition in HIV/AIDS Orphans in the Nyanza Province of Kenya: A comparison of Conventional Indices with a Composite Index of Anthropometric Failure (CIAF)", J Am Diet Assoc, 106: A20-A20

2. Bhattacharya AK (2000), "Assessment of growth and nutritional status in Indian population”, J Indian Anthropol Soc, 49: 69-102. 
3. Bhattacharya AK (2006), Composite index of anthropometric failure (CIAF) classification: is it more useful ? Bull WHO 86: 335.

4. Bose K Jr, Mandal GC, "Proposed new anthropometric indices of childhood undernutrition”. Malays J Nutr. 2010 Apr; 16(1):1316. Epub 2010 Apr 15. PubMed PMID: 22691860. [PubMed]

5. Dang SN \& Yan H (2007), “Optimistic factors affecting nutritional status among children during early childhood in rural areas of Western China". Zhonghua Yu Fang Yi Xue Za Zhi 41: 108-114.

6. Dewan D, Gupta R \&Kumar D. (2015), Can we rely solely on conventional measures to estimate undernutrition among underfives?,; 27, 3: 361-365.

7. Emina B.O.J. (2009) Child malnutrition in Cameroon: Does out-ofwedlock childbearing matter, African Population and Health Research Center, 17 p. Accessible sur: www.uclouvain.be/cps/ucl/doc/ demo/documents/Enima.pdf [consulté le 15 Janvier 2020]

8. Goswami M. (2016), "Prevalence of UnderNutrition Measured by Composite Index of Anthropometric Failure (CIAF) Among the Bhumij Children of Northern Odisha, India” J Nepal Paediatr Soc; 36 (1):61-67.

9. Harttgen K \& Misselhorn M. (2006), A multilevel approach to explain child mortality and undernutrition in South Asia and SubSaharan Africa. Available at website: www.opus.zbwkiel.de/volltexte/ 2006/4743/pdf/Misselhorn.pdf [accessed 15 Sept 2009].

10. Institut National de la Statistique (INS) et UNICEF. 2015. Enquête par grappes à indicateurs multiples, MICS6 République Démocratique du Congo 2017-2018, Rapport des principaux résultats. Kinshasa, R.D. Congo, INS et UNICEF.

11. Mahgoub S, Silo L \& Fields-Gardner C (2009), Effect of Dietary protein Enrichment Pilot Program on the growth of under-five children in day care centres in Gaborone, Botswana. Available at website: www.wishh.org/nutrition/ botswana_daycare_vasps.pdf [accessed 15 Sept 2009].

12. Mandal G. \& Bose K. (2009) “Assessment of Overall Prevalence of Undernutrition Using Composite Index of Anthropometric Failure (CIAF) among Preschool Children of West Bengal, India", Iranian Journal of Pediatrics, 2009; Vol 19 (No 3), Pp:237-243.

13. McDonald M. C. et al. (2013). "The effect of multiple anthropometric deficits on child mortality: meta-analysis of individual data in 10 prospective studies from developing countries". Am J Clin Nutr; 97:896-901. 
14. Ministère du Plan et Suivi de la Mise en œuvre de la Révolution de la Modernité (MPSMRM), Ministère de la Santé Publique (MSP) et ICF International, 2014. Enquête Démographique et de Santé en République Démocratique du Congo 2013-2014. Rockville, Maryland, USA: MPSMRM, MSP et ICF International.

15. Nandy S. M., Irving M, Gordon D, Subramanian SV \& Davey Smith G (2005). Poverty, child undernutrition and morbidity: new evidence from India. Bull World Health Organ 83: 210-216.

16. Nandy S. M., Miranda JJ (2008). "Overlooking undernutrition? Using a composite index of anthropometric failure to assess how underweight misses and misleads the assessment of undernutrition in young children". Soc Sci. Med.; 66 (9) : 1963-6. doi:10.1016/j.socscimed.2008.01.021. Epub 2008 Mar 4. PubMed PMID: 18299166; PubMed

Central PMCID: PMC2685640.[PubMed].

17. Nguemo N.C (2019). Facteurs explicatifs de la malnutrition chez les enfants de moins de 5 ans au Cameroun, Communication présentée à la réunion UEPA 2019, $23 \mathrm{p}$

18. OMS. Physical status: the use and interpretation of anthropometry. Report of a WHO Expert Committee. Technical Report Series No. 854. Geneva, World Health Organization, 1995. http://whqlibdoc.who.int/trs/WHO_TRS_854.pdf.

19. OMS. Training Course on Child Growth Assessment. Geneva, WHO, 2008.

20. Ramkuma S, VijayalakShmi S, kanagarajan P, Rajkuma P, Lokeshmaran A (2018). "Z-Score and CIAF-A Descriptive Measure to Determine Prevalence of Under-Nutrition in Rural School Children, Puducherry, India", Journal of Clinical and Diagnostic Research, 12(5): 24-27.

21. Seetharaman N, Chacko TV, Shankar SRL, Mathew AC (2007), "Measuring malnutrition - the role of Z-scores and the Composite Index of Anthropometric Failure (CIAF)" Indian J Comm Med 32: 35-9.

22. Sengupta P, Philip N, Benjamin AI (2010) ， "Epidemiological correlates of under nutrition in under 5 years children in an urban slum of Ludhiana" Health and Population Perspectives and Issues ; 33(1):1-9.

23. Shit S, Taraphdar P, Mukhopadhyay DK, Sinhababu A, Biswas AB. Assessment of nutritional status by composite index for anthropometric failure: A study among slum children in Bankura, West Bengal. Indian J Public Health [serial online] 2012; 56:305-7. Available from: http://www.ijph.in/text.asp?2012/56/4/305/106421. 
24. Svedberg P (2000), Poverty and Undernutrition; Theory, Measurement and Policy. Oxford India Paperbacks, New Delhi.

25. UNICEF (2019). La Situation des enfants dans le monde 2019. Enfants, nourriture et nutrition: Bien grandir dans un monde en mutation. UNICEF, New York.

26. Wamani H, Åstrøm AN, Peterson S, Tumwine JK, Tylleskär T (2007), Boys are more stunted than girls in sub-Saharan Africa: a meta-analysis of 16 demographic and health surveys. BMC Pediatr, 7(1): $1-0$. 